\title{
Die eigene Webpräsenz darf nicht fehlen
}

\section{Das Internet hat als Informationsmedium in der Medizin inzwischen einen enormen Stellenwert. Das gilt nicht nur für Ärzte, die das Web regelmäßig für berufliche Zwecke nutzen. Auch Patienten informieren sich zunehmend häu- figer über Krankheiten und Behandlungsangebote im Netz. Diese Entwicklung sollten Ärzte nutzen, um Patienten auf das Leistungsspektrum ihrer Praxis auf- merksam zu machen.}

- Ärzte nutzen das Internet zunehmend intensiver - so das Ergebnis der aktuellen LA-Med-Studie (Leseranalyse medizinische Fachkreise). Dabei halten sich die berufliche und private Nutzung in etwa die Waage. Favoriten bei der beruflichen Nutzung sind die medizinische Recherche, E-Mail, Online-Fortbildung (CME) und Datenaustausch.

Bei der Patientenkommunikation hingegen sind die Ärzte weniger aktiv. Dabei wächst das Bedürfnis der Patienten nach medizinischen Informa- tionen rasant, wie viele Studien zeigen: Immer mehr Patienten kommen mit selbst recherchierten Informationen in die Praxis. Das Problem dabei: Nach einer Prognos-Studie ist zum Beispiel weit mehr als die Hälfte der Informationen $\mathrm{zu}$ verschreibungspflichtigen Arzneimitteln falsch.

\section{Homepage erleichtert das Gespräch}

Für Ärzte ist es oft nicht einfach, gegen Fehlinformationen aus dem Web zu argumentieren. Um dem vorzubeugen, ist es hilfreich, selbst regelmäßig Informationen zu Krankheitsbildern bzw. den Angeboten der Praxis auf die eigene Homepage zu stellen. Oft lassen sich Patientengespräche dadurch einfacher gestalten. Hinzu kommt: Mehr als 50\% der Deutschen suchen im Internet nach Ärzten oder informieren sich vorab über Spezialisierungen, Öffnungszeiten etc.

\section{Hilfe bei Information und Marketing} Für die eigene Praxishomepage sprechen somit gleich zwei schlagende Argumente: Sie dient nicht nur dazu, Patienten auf zeitgemäße Weise objektiv zu informieren, sondern ist gleichzeitig ein zunehmend wichtiges Marketinginstrument.

GER/CP

\section{So schnell geht's ...}

\section{In wenigen Schritten zur professionellen Website: Tester gesucht!}

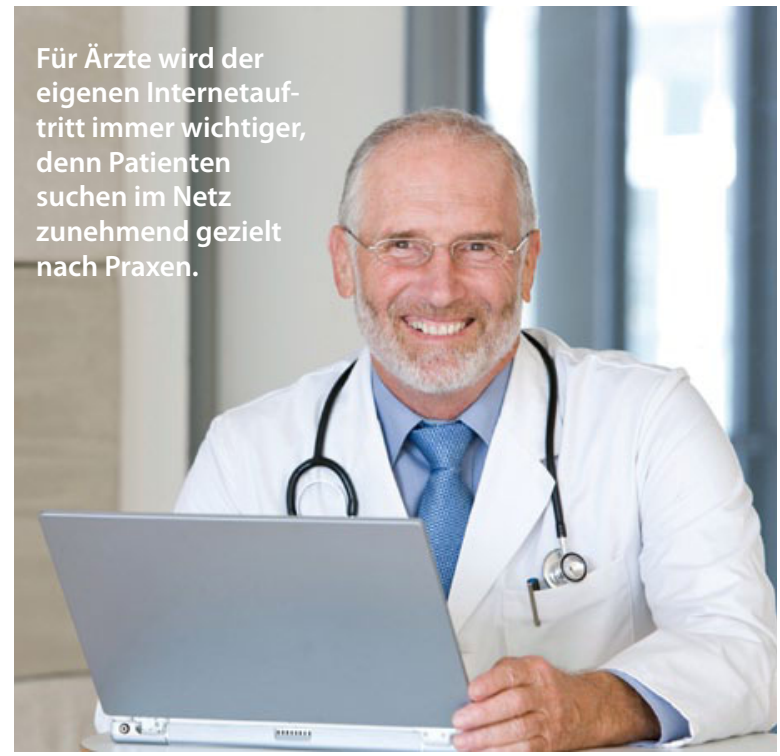

Ärzte, die noch keine eigene Internetpräsenz haben und nicht alles selber machen möchten, können jetzt das neue Angebot der Fachverlagsgruppe Springer Medizin, zu der auch die MMW gehört, nutzen.

Mit wenig Aufwand und zu geringen Kosten können Sie eine eigene Homepage aufbauen, die mehr als nur Standardinformationen zum Praxisteam und zu den Öffnungszeiten enthält. Der Zeitaufwand, um die eigene Website zu erstellen, ist absolut überschaubar und beträgt nur zirka 20 Minuten.

Ein weiterer Vorteil: Um Ihre Patienten auch aktuell zu informieren, können Sie auf Inhalte von Springer Medizin zurückgreifen.

Um dieses Angebot auf Herz und Nieren zu prüfen und den Service für die Online-Bedürfnisse optimal zu gestalten, werden jetzt 300 Testärzte gesucht, die als erste von dieser Möglichkeit profitieren wollen.

Unter den Testpersonen, die bis zum 30. November 2011 teilnehmen, werden zehn iPad 2 verlost!

Teilnahme: www.springermedizin.de/arztonline 